\title{
AÇÕES DE PROMOÇÃO DA SAÚDE REALIZADAS POR ENFERMEIROS NA AVALIAÇÃO DO CRESCIMENTO E DESENVOLVIMENTO INFANTIL ${ }^{1}$
}

\author{
NURSING HEALTH PROMOTION ACTIONS IN THE ASSESSMENT \\ OF CHILD GROWTH AND DEVELOPMENT
}

\author{
ACCIONES DE PROMOCIÓN DE LA SALUD REALIZADAS POR LAS \\ ENFERMERAS EN LA EVALUACIÓN DE CRECIMIENTO Y DESARROLLO \\ INFANTIL
}

\author{
Flávia Paula Magalhães Monteiro* \\ Thelma Leite de Araujo* \\ Lorena Barbosa Ximenes ${ }^{* * *}$ \\ Neiva Francenely Cunha Vieira***
}

\begin{abstract}
RESUMO
Objetivou-se identificar as ações de promoção da saúde pelo enfermeiro na avaliação do crescimento e do desenvolvimento infantil e analisá-las quanto às competências essenciais da promoção da saúde preconizadas pela Public Health Agency of Canada. Realizou-se uma Revisão integrativa em cinco bases de dados, utilizando uma combinação dos termos: crescimento e desenvolvimento, criança, promoção da saúde e enfermagem. Procedeuse a análise de 10 artigos na íntegra. Os estudos apontam que as ações de enfermagem são bem diversificadas e relacionam-se às medidas de investigação, avaliação e/ou intervenções, porém enfatizam apenas os aspectos nutricionais nesse processo, em detrimento às diferentes dimensões que contemplam o desenvolvimento infantil. Entre as sete competências identificadas, houve predomínio da avaliação e análise. Ademais, a competência diversidade e inclusão social foram identificadas em apenas dois estudos. Por fim, os enfermeiros vêem aplicando suas habilidades/competências na promoção da saúde, no entanto integram-nas sem utilizar um referencial teórico que norteie suas ações de crescimento e desenvolvimento infantil.
\end{abstract}

Palavras chave: Crescimento e desenvolvimento, promoção da saúde, enfermagem.

\begin{abstract}
Aimed to identify the actions of health promotion by nurses in the assessment of child growth and development and to analyze them as core competencies of health promotion recommended by the Public Health Agency of Canada. Carried out a integrative review of five databases, using a combination of the terms: growth and development, child health promotion and nursing. Proceeded to the analysis of articles 10 in its entirety. The

\footnotetext{
${ }^{1}$ Atividade desenvolvida na disciplina Análise Crítica das Práticas de Enfermagem na Promoção da Saúde do Programa de Pós-Graduação em Enfermagem da Universidade Federal do Ceará/UFC.

* Enfermeira. Aluna do curso de Doutorado em Enfermagem da Universidade Federal do Ceará (UFC), bolsista CNPq processo n¹41765/2010-0. Email: flaviapmm@yahoo.com.br

** Enfermeira. Docente do Programa de Pós-Graduação em Enfermagem da Universidade Federal do Ceará/UFC. Email: thelmaraujo2003@yahoo.com.br

${ }_{* * *}$ Enfermeira. Docente do Programa de Pós-Graduação em Enfermagem da Universidade Federal do Ceará/UFC. Email: lbximenes2005@uol.com.br

${ }^{* * * *}$ Enfermeira. Docente do Programa de Pós-Graduação em Enfermagem da Universidade Federal do Ceará/UFC. Email: neyvafrancenely@hotmail.com
} 
authors pointed out that nursing actions are well diversified and relate to measures of research, assessment and /or interventions, however they only emphasize on the nutritional aspects in this process, rather than the different dimensions that include child development. Among the seven competencies identified, evaluation and analysis were predominant. Moreover, the competence diversity and inclusion were identified in only two studies. Finally, the nurses see that their skills/competencies are being applied in health promotion, incorporating them without using a theoretical framework that guides their actions in child growth and development.

Key words: Growth and development, health promotion, nursing.

\section{RESUMEN}

El objetivo de este trabajo fue identificar las acciones para promoción de la salud por enfermeras en la evaluación del crecimiento y desarrollo del niño y analizar las competencias básicas de promoción de la salud recomendadas por la Agencia de Salud Pública de Canadá. Se llevó a cabo una revisión integradora en cinco bases de datos, utilizando una combinación de términos: crecimiento y desarrollo, niño, promoción de la salud y enfermería. Se procedió al análisis de los 10 artículos. Los autores señalaron que las Acciones de enfermería se encuentran diversificadas, y se refieren a las medidas de investigación, evaluación y/o intervenciones, sin embargo insisten en los aspectos nutricionales de este proceso, en lugar de las diferentes dimensiones que incluyen el desarrollo del niño. Entre las siete competencias identificadas, hubo predominio de la evaluación y análisis. Además, las competencias diversidad e inclusión social se identificaron en sólo dos estudios. Por último, las enfermeras ven aplicados sus conocimientos/competencias en promoción de la salud, sin utilizar un referencial teórico que oriente sus acciones en el crecimiento y desarrollo infantil.

Palabras clave: Crecimiento y desarrollo, promoción de la salud, enfermería.

Fecha recepción: 18/03/13 Fecha aceptación: 07/02/14

\section{INTRODUÇÃO}

A monitorização do crescimento foi recomendada desde a década de 70, na Conferência de Alma-Ata em 1978. No Brasil, em 1984, a criação do Programa de Assistência Integral à Saúde da Criança (PAISC) formulou um conjunto de ações básicas de saúde que visam à integralidade da assistência à saúde, com enfoque sobre ações de prevenção. Entre essas ações, encontrava-se o acompanhamento do crescimento e do desenvolvimento infantil por meio da Caderneta de Saúde da Criança elaborada e ampliada para abranger crianças com até dez anos de idade (1). Neste âmbito, a consulta de enfermagem incorpora ações de acompanhamento do crescimento e do desenvolvimento infantil, haja vista que a consulta compreende um modelo as- sistencial adequado às necessidades de saúde da criança e deve ser alicerçada nas etapas do processo de enfermagem.

O crescimento e o desenvolvimento configuram-se por ser global, dinâmico e contínuo e ocorre desde a concepção até o final da vida (2). Diante da complexidade, esse processo está sob efeito dos determinantes sociais, econômicos e culturais (3).

A avaliação do crescimento é um importante instrumento para acompanhamento das condições de saúde e nutrição, tanto de indivíduos quanto de populações. Na criança, a avaliação é realizada pela história clínica e social, exame físico, dados dos pais e a velocidade de crescimento. Avaliações em diferentes etapas da vida do ser humano em crescimento podem contribuir para explicações do que ocorreu em fases anteriores e sugerir riscos imediatos, mediatos e tardios para a 
saúde (4). Enquanto a avaliação do desenvolvimento é definida como processo de construção da identidade humana, que resulta da interação entre as influências biológicas, próprias da espécie e do indivíduo, e sua história de vida, contexto cultural e social (5).

Dessa forma, como ação básica de saúde, o acompanhamento do crescimento e do desenvolvimento é uma oportunidade do profissional de saúde analisar de forma integrada e preditiva a saúde da criança, com vista à ação resolutiva de promoção da saúde. Esta, definida como um processo social e político, não somente executado por ações envolvidas direcionadas ao fortalecimento de habilidades e capacidades individuais, mas também em ações que dizem respeito à mudança social, ambiente e condições econômicas. Tais como, aliviar o impacto sobre a saúde pública e individual, visto que se trata de um processo o qual permite às pessoas ampliar o controle dos determinantes de saúde e, portanto, melhorar sua saúde (6).

Com base nisso, a criança em seu processo evolutivo inicial apresenta um rápido e ordenado crescimento físico; e um desenvolvimento intelectual e social que necessita da abordagem e compreensão do enfermeiro para prestação de seus cuidados. Particularmente, essa avaliação vem sendo desempenhada na atenção primária em saúde, a qual inclui componentes essenciais: equidade, comunidade envolvida e participante, intersetorialidade, adequação de tecnologias e acessibilidade (7).

Os cuidados na atenção primária envolvem educação em saúde para crianças e comunidade; métodos de prevenção e controle de problemas identificados; promoção de adequada suplementação alimentar; garantia de instalações hídricas e sanitárias; cuidado em saúde materna e infantil incluindo o planejamento familiar; imunização; terapêutica apropriada para doenças comuns e o fornecimento de fármacos (8).

No âmbito da atenção primária de saú- de, o profissional desenvolve habilidades e competências que irão lhe respaldar no que concerne à avaliação do crescimento e do desenvolvimento infantil. Nesse sentido, competências são ações que abrangem conhecimento, habilidades e atitudes para a prática em saúde pública. São importantes por trancenderem os limites de disciplinas específicas, uma vez que fornecem o arcabouço para a prática eficaz na saúde pública.

Em face disso, a presente revisão teve como objetivo identificar as ações de promoção da saúde implementadas pelo enfermeiro na avaliação do crescimento e do desenvolvimento infantil e analisá-las quanto às competências essenciais da promoção da saúde preconizadas pela Public Health Agency of Canada, tais como: Ciências da saúde pública; Avaliação e análise; Políticas e programas de planejamento; Implementação e avaliação; Colaboração e apoio de parcerias; Diversidade e inclusão social; e Comunicação e liderança (9).

\section{MÉTODO}

Para o desenvolvimento deste estudo, realizou-se uma revisão integrativa da literatura no mês de outubro de 2010, de acordo com as seguintes etapas: definição de um problema; formulação de questões de pesquisa; estabelecimento de critérios de inclusão e exclusão; busca e categorização dos estudos; avaliação e análise dos dados, bem como a apresentação deles (10).

A etapa de formulação incluiu a clara identificação do problema de interesse, o propósito da revisão integrativa e a elaboração da pergunta norteadora. Com o intuito de direcionar a busca, elaborou-se a seguinte pergunta norteadora: Quais as ações de promoção da saúde desenvolvidas pelo enfermeiro na avaliação do crescimento e do desenvolvimento infantil? 
Para auxiliar o processo de análise da literatura, bem como minimizar vieses, construiu-se um protocolo, o qual contemplava em sua estrutura os seguintes aspectos: delimitação das bases de dados, objeto da pesquisa, estratégia para coleta de dados e para avaliação crítica e síntese dos dados (11). Além disso, os artigos foram avaliados quanto aos níveis de evidência I a VII propostos por Melnyk e Fineout-Overholt (12), os quais descrevem: nível I para revisões sistemáticas/diretrizes clínicas; nível II estudos clínicos randomizados controlados (ECRC) bem delineados; nível III evidências extraídas de ensaios clínicos bem delineados sem randomização; nível IV estudos retrospectivos bem delineados/estudos de coorte bem delineados/caso-controle; nível $\mathrm{V}$ revisões sistemáticas de estudos descritivos; nível VI estudos epidemiológicos/transversal, descritivos e qualitativos e nível VII revisões da literatura nas quais se obtém a opinião de especialistas.

Com relação aos critérios de inclusão, estabeleceram-se: artigos científicos que apresentassem resumo, além de texto completo, disponíveis eletronicamente nas bases de dados ou no periódico do portal da Coordenação de Aperfeiçoamento de Pessoal de Nível Superior (CAPES), nos idiomas inglês, espanhol ou português, publicados até outubro de 2010, os quais abordassem a temática de interesse. Além disso, a busca restrigiu-se às produções que envolvessem ações de promoção da saúde que retratassem a avaliação do crescimento e do desenvolvimento que tivessem pelo menos um dos autores enfermeiro. Os critérios de exclusão foram estabelecidos em: publicações do tipo editorial, resumo em anais de evento, revisões narrativas e sistemáticas, estudos de reflexão, dissertação e tese, bem como publicações repetidas.

No concernente à seleção da literatura, foi realizada de forma ordenada nas bases de dados Scopus, Pubmed (National Library of
Medicine), Cumulative Index to Nursing and Allied Health Literature (CINAHL with full text), Centro Latino-Americano e do Caribe de Informações em Ciências da Saúde (LILACS) e The Cochrane Library, considerandose as especificidades de cada uma.

A busca de estudos nas bases de dados foi realizada mediante a identificação dos descritores no Medical Subject Headings (MeSH) e nos Descritores em Ciências da Saúde (DeCS), considerando-se os termos presentes na pergunta norteadora. Assim, utilizaram-se uma combinação dos termos: Crescimento e desenvolvimento; criança; promoção da saúde; enfermagem, respectivamente nas línguas portuguesas, inglesas e espanholas.

Todos os artigos identificados foram submetidos às duas etapas de avaliação. A primeira dirigia-se à apreciação do título e do resumo, com vistas a identificar sua adequação à pergunta norteadora e aos critérios de inclusão e exclusão pré-estabelecidos. Em seguida, a segunda etapa compreendeu a análise dos artigos quanto ao ano de publicação, tipo de estudo e país onde foi realizado, objeto, idade das crianças e ações de promoção da saúde. Por fim, as competências essenciais em promoção da saúde foram discutidas nas sete categorias numeradas em: $1^{\text {a }}$ ) Ciências da saúde pública; $2^{\mathrm{a}}$ ) Avaliação e análise; 3a) Políticas e programas de planejamento; 4a) Implementação e avaliação; 5a) Colaboração e apoio de parcerias; 6a) Diversidade e inclusão social; 7a) Comunicação e liderança (9).

Além disso, os artigos foram analisados quanto ao objeto de estudo, idade da criança avaliada pelo enfermeiro durantes as consultas referentes ao crescimento e desenvolvimento, tipo de estudo e ações de enfermagem realizadas durantes tais consultas.

A seguir, na Figura 1 tem-se um demonstrativo ilustrativo sobre a busca e seleção das publicações analisadas. 
Figura 1. Demonstrativo ilustrativo sobre a busca e seleção das publicações analisadas.

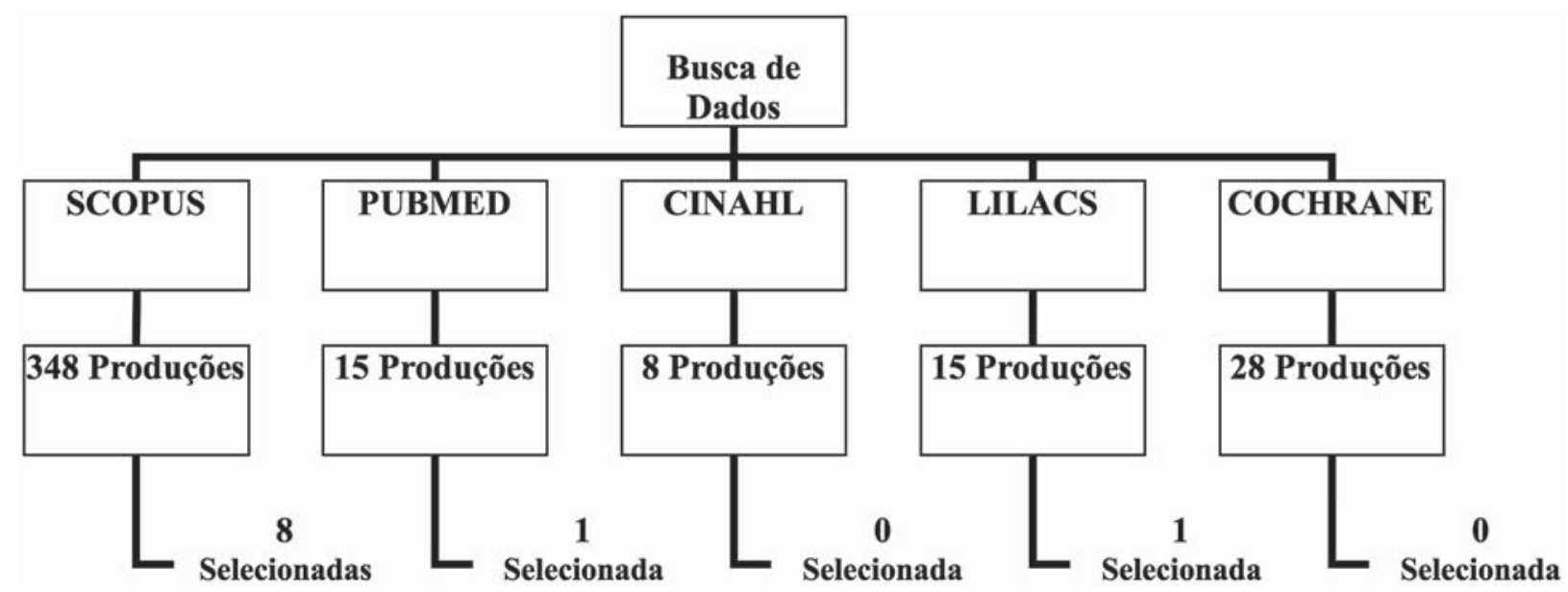

\section{RESULTADOS}

Dessa forma, procedeu-se a análise de 10 artigos na íntegra publicados entre 2003 e 2010, os quais estavam distribuídos: oito deles na base de dados Scopus, 1 artigo nas bases de dados Pubmed e Lilacs, respectivamente (Tabela 1). Por outro lado, as produções analisadas nas bases de dados Cinahl e Cochrane foram excluídas, de acordo com os critérios previamente estabelecidos.

Entre as publicações, observou-se o predomínio de estudos descritivos (5), prospectivos e longitudinais (3) e Ensaios Clínicos Controlados Randomizados (2), os quais apresentaram níveis de evidência variando entre VI, IV e II, respectivamente. A maioria foi desenvolvida na América do Norte, seguida pela produção nos países Asiáticos e América do Sul.

As publicações apresentaram diversas temáticas e ações de promoção da saúde nos mais variados contextos da saúde e faixas etárias das crianças, incluindo desde os 2 meses de vida até os 19 anos, o que demonstra relativa complexidade das ações envolvidas.
Como foram observadas, as ações de enfermagem estão relacionadas às medidas de investigação, avaliação e/ou intervenções que abordam diferentes aspectos do crescimento e desenvolvimento infantil. No geral, as ações enfatizam a avaliação nutricional da criança, especificamente sobre desordens nutricionais: desnutrição, sobrepeso ou obesidade, que alteram as dimensões corporais indicadas pelas medidas de peso e comprimento. Em contrapartida, a literatura aponta que o desenvolvimento infantil é mais abrangente, visto que inclui o próprio crescimento infantil, e corresponde às alterações da composição e do funcionamento das células, à dimensão dos membros, à maturação dos órgãos e à aquisição de novas funções e está caracterizado pelo aumento da capacidade do indivíduo na realização de funções complexas (3).

No geral, as ações de promoção da saúde no acompanhamento do crescimento e desenvolvimento infantil identificadas nos estudos são mais pontuais e se referem à ava- 
liação antropométrica, registros alimentares (aleitamento materno), avaliação do histórico de imunização, retratando procedimentos desenvolvidos durante as consultas de enfermagem. Neste âmbito, o papel dos profissionais depende de treinamento, organização, experiência pessoal e interesse sobre os propósitos da saúde pública em melhorar o estilo de vida das crianças e de seus familiares.

Por conseguinte, surgem lacunas no cuidado que priorizam alguns procedimentos técnicos, em detrimento às complexas dimensões do crescimento e desenvolvimento infantil, tendo em vista que as melhorias em saúde não podem ser dirigidas por serviços individuais, mas o agrupamento de ações ambientais e econômicas, pautadas sobre os determinantes de saúde (13).

Por outro lado, outras ações concernentes ao empoderamento das famílias acerca da condição de saúde/doença dos seus filhos, nas quais se observam interações educativas entre enfermeiros, familiares e crianças, apresentam, mesmo que tímida, uma preocupação com abordagem da promoção da saúde em suas ações. De acordo com a carta de Ottawa (6) os pilares da promoção da saúde se erguem sobre o empoderamento de pessoas para conseguir o controle da sua própria saúde e incluir mudanças de estratégias para fortalecer comunidades, desenvolver ambientes sustentáveis, informar e educar sobre as questões de saúde. Em face disso, os enfermeiros têm empoderado familiares e crianças, tornando-as capazes de perceber seus problemas e tomar decisões que afetarão sua própria saúde, principalmente relacionadas ao crescimento e desenvolvimento infantil.

Por outro lado, alguns estudos, em síntese, evidenciaram aspectos sobre o desenvolvimento físico, motor, cognitivo, mental e psicossocial, de forma menos expressiva, o que demonstra uma fragilizada atuação de enfermagem sobre tais aspectos. Possivelmente, o desenvolvimento infantil abrange diferentes facetas que podem até mesmo incluir o pró- prio crescimento. Em virtude disso, tem-se observado que a literatura ainda apresenta divergências sobre os conceitos de crescimento e desenvolvimento infantil e, por conseguinte, gera disparidade de opiniões entre os próprios profissionais da saúde.

No geral, por abranger diferentes aspectos sobre o crescimento e desenvolvimento infantil, as ações de promoção da saúde analisadas são complementares. Dessa forma, sugere-se particularidades delas para sua implementação na assistência de enfermagem, englobando os determinantes do adequado crescimento e desenvolvimento infantil em consonância com os preceitos preconizados pelas competências e habilidades essenciais na promoção da saúde.

Nesse sentido, o enfermeiro atuante sobre o cuidado primário à criança, inicialmente deve investigar determinantes sociais, econômicos e de saúde na tentativa de realizar um diagnóstico situacional da família da criança e do meio onde esta se insere e direcionar os cuidados previstos. A partir disso, informações/orientações e supervisões são delineadas com base nas possibilidades e limitações da família, resgatando a necessidade de educá-la/ensiná-la para o autocuidado, na tentativa de torná-la co-responsável (empoderada) por suas decisões sobre a saúde.

Ademais, o enfermeiro torna-se um mediador entre o serviço de saúde e a comunidade, atento às necessidades das crianças durante as visitas domiciliares. Dentro desta perspectiva, o profissional estabelece os determinantes de saúde que compõem a avaliação do crescimento, por meio da antropometria de forma periódica e intervencionista e a avaliação do desenvolvimento infantil perpassa pelo processo de evolução física, cognitiva, motora, psicossocial e mental da criança.

A seguir, são descritas as discussões sobre competências essenciais na promoção da saúde (9) segundo sua maior e menor abrangência, respectivamente nos artigos ora analisados. 
Todos os artigos analisados evidenciaram de forma explícita ou não, ações de enfermagem associadas ao crescimento e desenvolvimento infantil, referentes à competência "Avaliação e Análise" a qual tem o intuito de coletar, avaliar, analisar e aplicar as informações (incluindo dados, fatos, conceitos e teorias), necessárias para tomada de decisões baseadas em evidências, bem como elaborar orçamentos e relatórios, realizar investigações e recomendar ações específicas com base na análise de informações para políticas e programas (9).

Especificamente, as ações abordaram os níveis de empoderamento de famílias cujos filhos apresentavam alterações no desenvolvimento, na tentativa de identificar se os familiares dispunham de atitude, conhecimento para controlar as situações adversas da criança no próprio ambiente de cuidado domiciliar, articulando o serviço de saúde e a comunidade (14).

Além disso, outro aspecto encontrado nos estudos (15) refere-se à investigação do conhecimento de pais/familiares acerca da alimentação de suas crianças, considerando a qualidade e a quantidades de alimentos complementares, sobretudo ao analisar no ambiente domiciliar o preparo e as condições dos alimentos. Nesse estudo, avaliavase, até mesmo, a prática da amamentação, informando sobre a técnica correta, benefícios e, resgatando comparações com taxas de amamentação e/ou desmame precoce em relatórios nacionais e pesquisas, aliando-os a investigação de medidas antropométricas e de outros determinantes de saúde, tais como avaliação da pressão arterial, escolaridade materna e habilidades cognitivas da criança, como benefícios advindos do aleitamento materno (16).

Para efetiva execução e aprendizado das informações repassadas pelos enfermeiros no acompanhamento do crescimento e desenvolvimento infantil, o profissional insere-se no âmbito familiar com o intutito de resgatar pensamentos e sentimentos acerca da necessidade iminente de modificar comportamentos e atitudes negativas de saúde, especificamente relacionadas ao sobrepeso e à obesidade, para então promover comportamentos saudáveis (17).

Particularmente, em relação aos comportamentos cognitivos conforme citam os pesquisadores (18), nessa situação, o profissional reconhece pensamentos e sentimentos referentes à obesidade infantil e tenta resgatar problemas os quais serão determinantes para incitar diálogos que guiem a auto-reflexão dos familiares, no sentido de tomar suas próprias decisões. Nesta situação, observamse que as ações de enfermagem estão embasadas no conhecimento teórico-científico, embora não esteja explícito o uso de referenciais teóricos da promoção da saúde.

Ademais, sete estudos (15-21) mencionaram ações de enfermagem contempladas na competência "Planejamento Implementação e Avaliação de Programas e Políticas”- competências necessárias para efetivamente escolher opções, planejar, implementar e avaliar políticas e/ou programas na saúde pública.

Essa competência demonstra habilidades no cenário e acompanhamento de prioridades para maximizar resultados baseados na avaliação de recursos (9), por meio de treinamentos sobre modificações alimentares entre indivíduos e grupos; discussão de estratégias para aumentar a atividade física; resolução de problemas e barreiras para reduzir o peso; melhoria do consumo alimentar por meio de demonstração das habilidades dos familiares, bem como avaliação da interação mãe-criança nas habilidades motoras finas.

Nesse contexto, as ações identificadas demonstram que a promoção da saúde vem sendo trabalhada nos diferentes cenários da comunidade, entre eles a escola, ambiente domiciliar e o próprio serviço de saúde. Para isso, variadas estratégias são aplicadas com o intuito de incentivar escolhas saudáveis em saúde por meio de tecnologias e, até mesmo, pelo contato direto entre mãe e filho.

Em face disso, observam-se também que 
essas ações de enfermagem tentam incluir os pais/cuidadores no planejamento e execução de tarefas que, possivelmente, poderão influenciar positivamente na mudança de comportamento, no incremento de atividades físicas e no hábito alimentar (17). Além disso, a colaboração e o diálogo com familiares na criação de objetivos individuais e estratégias para sobrepor barreiras irão proporcionar o empoderamento das famílias e, por conseguinte, continuidade de ações de saúde.

Outra competência abordada em sete estudos (14-23) foi a "comunicação", incluindo dimensões desta, tais como as trocas internas e externas, por escrito, verbal, não-verbal e habilidades de escuta, fornecendo informações adequadas aos diferentes públicos, na tentativa de mobilizá-los, utilizando meios adequados (9).

As ações se davam, especificamente, quando os enfermeiros repassavam informações sobre o crescimento e desenvolvimento infantil. Entre elas, orientações sobre a alimentação complementar, sinais de desnutrição e qualidade/quantidade de alimentos e, também, diálogos/interação entre mães e crianças no acompanhamento de habilidades motoras finas.

Particularmente, o enfermeiro exerce importante papel fundamental em comunicar no acompanhamento do crescimento e desenvolvimento infantil, sempre retomando aspectos na evolução da criança à medida que ocorre o processo de maturação de órgãos/sistemas corporais. Para isso, o profissional faz uso de registros os quais facilitam a comunicação, também, entre outros profissionais. Nesse contexto, a capacidade de escuta, aliada ao desempenho clínico dos profissionais e à interação são fatores que podem influenciar a forma como os usuários vinculam-se à unidade de saúde, especialmente dos cuidadores das crianças.

Por fim, apenas duas competências foram menos abordadas nas ações de promoção da saúde desenvolvidas pelos enfermeiros na sua prática assistencial: "Liderança" e "Diversidade e inclusão social".

Possivelmente, o menor enfoque direcionado a essas competências tenha sido de certa forma negligenciado, tendo em vista que os enfermeiros têm assumido papéis de liderança nos diversos âmbitos da saúde, sobretudo durante o acompanhamento do crescimento e do desenvolvimento de crianças.

No entanto, para essas ações, são necessárias habilidades para transformar os cuidadores em pessoas ativas ao acompanhar o crescimento e o desenvolvimento infantil, visto que, na promoção da saúde dessas crianças, o enfermeiro tem a constante tarefa de informar sobre as necessidades, para que elas e seus familiares sejam capazes de reconhecer alterações e assumir a função de coordenadores, negociando com os profissionais e administradores locais o bem-estar e a independência de seus filhos.

Por outro lado, no que se refere à competência "diversidade e inclusão social", os estudos (22) mencionaram ações de enfermagem no crescimento e no desenvolvimento de crianças de origem estrangeira. Estas ações são pouco desenvolvidas, principalmente porque os enfermeiros têm encontrado barreiras culturais, na linguagem e, também, falta de recursos de infra-estrutura (22). Isto acontece embora seja necessário ao enfermeiro conhecer as diferenças culturais, no que concerne à alimentação, linguagem, hábitos de vida, acessibilidade ao serviço de saúde (recursos), situação de saúde desse grupo específico, políticas públicas para o setor da saúde, para então promover habilidades na comunicação/interação com crianças e familiares e delinear estratégias durante visitas domiciliares ou no próprio serviço de saúde.

No geral, as sete competências essenciais na promoção da saúde foram identificadas entre as ações de enfermagem, sendo 
que houve predomínio de ações referentes à avaliação e análise, denotando considerada preocupação dos enfermeiros em identificar e aplicar informações para conduzir investigações, bem como realizar recomendações políticas e promover o desenvolvimento de programas na saúde da criança.

Em contrapartida, a competência denominada diversidade e inclusão social foi identificada em apenas dois estudos, sugerindo que o enfermeiro estenda suas ações de promoção nos diferentes contextos da saúde, de acordo com os princípios na declaração de Jacarta. Nesta, a promoção da saúde atua sobre os determinantes da saúde na tentativa de criar o maior benefício para todos e contribuir de maneira significativa para a redução das iniqüidades nas questões de saúde, para assegurar os direitos humanos.

Com base no exposto, destacam-se que os enfermeiros vêem aplicando suas habilidades/competências na promoção da saúde, não obstante integram-nas sem utilizar um referencial teórico que norteie suas ações no complexo processo de crescimento e desenvolvimento infantil, tendo em vista a vigente disparidade de conceitos, subcategorias e termos utilizados sobre tais fenômenos.

Tabela 1. Caracterização das publicações quanto ao objeto e tipo de estudo, idade da criança, ações de promoção da saúde na avaliação do crescimento e do desenvolvimento infantil, tipo e local do estudo, as competências identificadas nas bases de dados e os níveis de evidências. Novembro, 2010.

\begin{tabular}{|c|c|c|c|c|c|c|}
\hline Artigo & Objeto de estudo & $\begin{array}{c}\text { Idade } \\
\text { da } \\
\text { criança }\end{array}$ & $\begin{array}{c}\text { Ações de Enferma- } \\
\text { gem na Promoção } \\
\text { da saúde }\end{array}$ & $\begin{array}{c}\text { Tipo de } \\
\text { Estudo/País }\end{array}$ & $\begin{array}{l}\text { Compe- } \\
\text { tências }\end{array}$ & $\begin{array}{l}\text { Níveis de } \\
\text { evidência }\end{array}$ \\
\hline $\begin{array}{l}\text { Wakimizu R, Fu- } \\
\text { jioka H, Yoneyama } \\
\text { A. Empowerment } \\
\text { process for families } \\
\text { rearing children } \\
\text { with development } \\
\text { disorders in Japan. } \\
\text { Nurs Health Sci. } \\
\text { 2010; 12: 322-328 }\end{array}$ & $\begin{array}{l}\text { Empoderamento } \\
\text { de famílias com } \\
\text { crianças acometi- } \\
\text { das por desordens } \\
\text { no desenvolvi- } \\
\text { mento }\end{array}$ & $\begin{array}{l}5 \text { a } 19 \\
\text { anos }\end{array}$ & $\begin{array}{l}\text { 1) Avaliação e } \\
\text { análise dos níveis } \\
\text { e expressões de } \\
\text { empoderamento na } \\
\text { família de crianças } \\
\text { com desordens no } \\
\text { desenvolvimento, } \\
\text { no serviço de saúde } \\
\text { e na comunidade; } \\
\text { 2) Colaboração } \\
\text { com profissionais } \\
\text { do cuidado/educa- } \\
\text { cionais e parcerias } \\
\text { com a adminis- } \\
\text { tração local; } \\
\text { 3) Promoção da } \\
\text { liderança de pais } \\
\text { frente à problemá- } \\
\text { tica das crianças. }\end{array}$ & $\begin{array}{l}\text { Descritivo/ } \\
\text { Japão }\end{array}$ & $2,4,6$, e 7 & VI \\
\hline
\end{tabular}


Continuação Tabela 1.

\begin{tabular}{|c|c|c|c|c|c|c|}
\hline $\begin{array}{l}\text { Shi L et al. Effecti- } \\
\text { veness of an educa- } \\
\text { tional intervention } \\
\text { on complementary } \\
\text { feeding practices } \\
\text { and growth in rural } \\
\text { China: a cluster } \\
\text { randomized con- } \\
\text { trolled trial. Public } \\
\text { Health Nutr. } 2009 \text {; } \\
\text { 13(4): 556-565 }\end{array}$ & $\begin{array}{l}\text { Avaliação de } \\
\text { uma intervenção } \\
\text { educacional para } \\
\text { melhorar a prática } \\
\text { de alimentação } \\
\text { complementar, a } \\
\text { saúde nutricional } \\
\text { da criança e o cres- } \\
\text { cimento. }\end{array}$ & $\begin{array}{c}2 \text { a } 4 \\
\text { meses }\end{array}$ & $\begin{array}{l}\text { 1) Avaliação e } \\
\text { análise do con- } \\
\text { hecimento sobre } \\
\text { alimentação das } \\
\text { crianças, prevalên- } \\
\text { cia de desnutrição } \\
\text { e morbi-mortali- } \\
\text { dade; } \\
\text { 2)Fornecimento de } \\
\text { Informações sobre } \\
\text { alimentação com- } \\
\text { plementar, sinais de } \\
\text { desnutrição e qua- } \\
\text { lidade/quantidade } \\
\text { de alimentos; } \\
\text { 3) Implementação } \\
\text { de treinamentos } \\
\text { sobre os problemas } \\
\text { identificados e con- } \\
\text { selhos individuais e } \\
\text { grupais; } \\
\text { 4) Envolvimento } \\
\text { de parcerias, como } \\
\text { líder comunitário, } \\
\text { familiares e profis- } \\
\text { sionais na estraté- } \\
\text { gia educacional. }\end{array}$ & $\begin{array}{l}\text { Ensaio clíni- } \\
\text { co controla- } \\
\text { do randomi- } \\
\text { zado/China }\end{array}$ & $\begin{array}{c}1,2,3,4 \\
\text { e } 6\end{array}$ & II \\
\hline $\begin{array}{l}\text { Kramer MS et al. } \\
\text { Health and develo- } \\
\text { pment outcomes in } \\
6.5-\text { y-old children } \\
\text { breastfed exclusi- } \\
\text { vely for } 3 \text { or } 6 \text { mo. } \\
\text { Am J Clin Nutr. } \\
2009 \text {; } 90: 1070-4{ }^{(x)}\end{array}$ & $\begin{array}{l}\text { Avaliação da } \\
\text { saúde e do des- } \\
\text { envolvimento de } \\
\text { crianças que foram } \\
\text { amamentadas ex- } \\
\text { clusivamente por } \\
3 \text { meses e por } 6 \\
\text { meses. }\end{array}$ & 6,5 anos & $\begin{array}{l}\text { 1) Avaliar o es- } \\
\text { tado de saúde de } \\
\text { crianças amamen- } \\
\text { tadas exclusiva- } \\
\text { mente durante } 3 \\
\text { meses e comparar } \\
\text { com aquelas ama- } \\
\text { mentadas parcial- } \\
\text { mente durante } 6 \\
\text { meses; } \\
\text { 2) Realização de } \\
\text { visitas domiciliares } \\
\text { para verificar medi- } \\
\text { das antropométri- } \\
\text { cas, pressão arterial } \\
\text { das crianças, esco- } \\
\text { laridade materna, } \\
\text { habilidade cogni- } \\
\text { tiva, com vista à } \\
\text { aplicação de deter- } \\
\text { minantes de saúde. }\end{array}$ & $\begin{array}{l}\text { Estudo } \\
\text { Prospectivo } \\
\text { de coorte/ } \\
\text { Canadá }\end{array}$ & 2,3 & VI \\
\hline
\end{tabular}


Continuação Tabela 1.

\begin{tabular}{|c|c|c|c|c|c|c|}
\hline $\begin{array}{l}\text { Tyler DO, Horner } \\
\text { SD. Collaborating } \\
\text { with low-income } \\
\text { families and their } \\
\text { overweight chil- } \\
\text { dren to improve } \\
\text { weigth-related } \\
\text { behaviors; an in- } \\
\text { tervention process } \\
\text { evaluation. J Spec } \\
\text { Pediatr Nurs. 2008; } \\
\text { 13(4): 263-274 }{ }^{(\mathrm{x})}\end{array}$ & $\begin{array}{l}\text { Avaliação de } \\
\text { crianças com obe- } \\
\text { sidade ou sobre- } \\
\text { peso no ambiente } \\
\text { escolar. }\end{array}$ & 8 a 12 anos & $\begin{array}{l}\text { 1)Avaliação de } \\
\text { percepços e sen- } \\
\text { timentos sobre a } \\
\text { mudança de roti- } \\
\text { na da família da } \\
\text { criança com obesi- } \\
\text { dade ou sobrepeso } \\
\text { durante visitas } \\
\text { domiciliares; } \\
\text { 2)Discussão e } \\
\text { análise de proble- } \\
\text { mas: alimentares, } \\
\text { sedentarismo pelos } \\
\text { próprios familiares } \\
\text { para promoção da } \\
\text { mudança de com- } \\
\text { portamento; } \\
\text { 3)Orientação e dis- } \\
\text { cussão de estraté- } \\
\text { gias para aumentar } \\
\text { a atividade física, } \\
\text { melhorar o consu- } \\
\text { mo alimentar por } \\
\text { meio de demons- } \\
\text { tração das habilida- } \\
\text { des dos familiares, } \\
\text { buscando adequar } \\
\text { suas possibilidades } \\
\text { e limitações. }\end{array}$ & $\begin{array}{l}\text { Longitudi- } \\
\text { nal/EUA }\end{array}$ & $\begin{array}{l}1,2,3,4 \\
\quad 6 \text { e } 7\end{array}$ & VI \\
\hline $\begin{array}{l}\text { Berry et al. An } \\
\text { intervention for } \\
\text { multiethnic obe- } \\
\text { se parents and } \\
\text { overweight chil- } \\
\text { dren. Appl Nurs } \\
\text { Res. 2007; 20: 63- } \\
71{ }^{(\mathrm{x})}\end{array}$ & $\begin{array}{l}\text { Intervenções usan- } \\
\text { do habilidades de } \\
\text { enfrentamento } \\
\text { para pais obesos e } \\
\text { crianças com so- } \\
\text { brepeso atendidas } \\
\text { em um programa } \\
\text { de controle do } \\
\text { peso. }\end{array}$ & 7 a 17 anos & $\begin{array}{l}\text { 1)Avaliação e } \\
\text { transmissão de } \\
\text { informações sobre } \\
\text { educação nutricio- } \\
\text { nal e exercícios; } \\
\text { 2)Parcerias e co- } \\
\text { laboração entre } \\
\text { pais e crianças para } \\
\text { modificaçoes no } \\
\text { comportamento } \\
\text { sobre a melhora } \\
\text { da auto-estima e } \\
\text { aprendizado de } \\
\text { novas habilidades } \\
\text { para controlar o } \\
\text { estresse; } \\
\text { 3)Implementação } \\
\text { de estratégia de } \\
\text { ensino incluindo } \\
\text { modificações no } \\
\text { comportamento } \\
\text { cognitivo; reso- } \\
\text { lução de problemas } \\
\text { e barreiras para } \\
\text { reduzir o peso; } \\
\text { motivação e reso- } \\
\text { lução de conflitos } \\
\text { internos. }\end{array}$ & $\begin{array}{l}\text { Estudo Pilo- } \\
\text { to - Ensaio } \\
\text { Clínico Ran- } \\
\text { domizado/ } \\
\text { EUA }\end{array}$ & $2,3,4$ e 6 & II \\
\hline
\end{tabular}


Continuação Tabela 1.

\begin{tabular}{|c|c|c|c|c|c|c|}
\hline $\begin{array}{l}\text { Reifsnider E, Keller } \\
\text { CS, Gallagher M. } \\
\text { Factors related to } \\
\text { overweight and risk } \\
\text { for overweight sta- } \\
\text { tus among low-in- } \\
\text { come Hispanic } \\
\text { children. J Pediatr } \\
\text { Nurs. } 2006 ; 21(3) \text { : } \\
186-197^{(\mathrm{x})}\end{array}$ & $\begin{array}{l}\text { Investigação de } \\
\text { fatores relaciona- } \\
\text { dos entre o sobre- } \\
\text { peso/obesidade, a } \\
\text { criança e o ambien- } \\
\text { te em um programa } \\
\text { de nutrição. }\end{array}$ & $\begin{array}{c}\text { Entre } 1 \text { a } 2 \\
\text { anos. }\end{array}$ & $\begin{array}{l}\text { 1) Avaliação e aná- } \\
\text { lise dos indicadores } \\
\text { nutricionais: me- } \\
\text { didas antropomé- } \\
\text { tricas, história da } \\
\text { alimentação (inci- } \\
\text { dência e duração } \\
\text { da amamentação); } \\
\text { disponibilidade } \\
\text { de alimento e se- } \\
\text { gurança alimentar } \\
\text { para a família e } \\
\text { ocupação da mãe; } \\
\text { 2)Avaliação da } \\
\text { interação mãe- } \\
\text { criança nas habi- } \\
\text { lidades motoras } \\
\text { finas. }\end{array}$ & $\begin{array}{l}\text { Estudo Lon- } \\
\text { gitudinal/ } \\
\text { EUA }\end{array}$ & 1,2 e 3 & VI \\
\hline $\begin{array}{l}\text { Kilanowski JF, } \\
\text { Ryan-Wenger N. } \\
\text { Health status in an } \\
\text { invisible popula- } \\
\text { tion: carnival and } \\
\text { migrant worked } \\
\text { children. West J } \\
\text { Nurs Res. 2007; } \\
29(1): 100-120^{(\mathrm{x})}\end{array}$ & $\begin{array}{l}\text { Avaliação do es- } \\
\text { tado de saúde de } \\
\text { crianças imigrantes. }\end{array}$ & Até 1 ano. & $\begin{array}{l}\text { 1) Investigação, } \\
\text { análise e avaliação } \\
\text { de indicadores de } \\
\text { saúde: estado de } \\
\text { imunização, saúde } \\
\text { dental e parâmetros } \\
\text { de crescimento: } \\
\text { peso, comprimen- } \\
\text { to/altura e circun- } \\
\text { ferência cefálica } \\
\text { das crianças imi- } \\
\text { grantes; }\end{array}$ & $\begin{array}{l}\text { Descriti- } \\
\text { vo-explora- } \\
\text { tório/E }\end{array}$ & $1,2,3$ e 5 & VI \\
\hline $\begin{array}{l}\text { Peña YF, Almeida } \\
\text { MCP, Duranza } \\
\text { RLC. The nursing } \\
\text { work process in } \\
\text { care for healthy } \\
\text { children at a social } \\
\text { security institution } \\
\text { in Mexico. Rev Lat } \\
\text { Am Enfermagem. } \\
2006 ; 14(5): 651- \\
7^{(y)}\end{array}$ & $\begin{array}{l}\text { Processo de trabal- } \\
\text { ho da enfermeira } \\
\text { na nutrição e no } \\
\text { crescimento e } \\
\text { desenvolvimento } \\
\text { infantil. }\end{array}$ & Até 5 anos & $\begin{array}{l}\text { 1)Visitas domicilia- } \\
\text { res para avaliação } \\
\text { das medidas antro- } \\
\text { pométricas; } \\
\text { 2) Diálogo e inte- } \\
\text { rações entre pro- } \\
\text { fissionais, mães e } \\
\text { crianças; } \\
\text { 3) Avaliação do } \\
\text { cartão de vacinas; } \\
\text { 4) Registro da } \\
\text { criança para refe- } \\
\text { renciá-la à saúde } \\
\text { dental e vacinação. }\end{array}$ & $\begin{array}{l}\text { Estudo } \\
\text { descritivo / } \\
\text { México }\end{array}$ & 2,3 e 6 & VI \\
\hline
\end{tabular}


Continuação Tabela 1.

\begin{tabular}{|c|c|c|c|c|c|c|}
\hline $\begin{array}{l}\text { Berlin A, Hylan- } \\
\text { der I, Törnkvist } \\
\text { L. Primary child } \\
\text { health care nurses' } \\
\text { assessment of heal- } \\
\text { th risks in children } \\
\text { of foreing origin } \\
\text { and their parent - a } \\
\text { theoretical model. } \\
\text { Scand J Caring Sci. } \\
\text { 2008; 22: 118-127 }\end{array}$ & $\begin{array}{l}\text { Avaliação de ris- } \\
\text { co de saúde de } \\
\text { crianças estrangei- } \\
\text { ras por enfermeiras } \\
\text { na Suécia. }\end{array}$ & - & $\begin{array}{l}\text { 1)Avaliação do } \\
\text { desenvolvimen- } \\
\text { to físico, motor, } \\
\text { cognitivo, mental e } \\
\text { psicossocial; } \\
\text { 2)Realização de } \\
\text { visitas domiciliares } \\
\text { para promoção do } \\
\text { contato familiar; } \\
\text { 3)Avaliação do am- } \\
\text { biente domiciliar; } \\
\text { 4)Colaboração de } \\
\text { pais e crianças, } \\
\text { resgatando com- } \\
\text { portamentos, } \\
\text { controle do peso e } \\
\text { comprimento. }\end{array}$ & $\begin{array}{l}\text { Descritivo/ } \\
\text { Suécia }\end{array}$ & $2,4,5$ e 6 & VI \\
\hline $\begin{array}{l}\text { Figueiredo GLA, } \\
\text { Mello DF. A prática } \\
\text { de enfermagem na } \\
\text { atenção à saúde da } \\
\text { criança em unidade } \\
\text { básica de saúde. } \\
\text { Rev Lat Am Enfer- } \\
\text { magem } 2003 ; 11 \\
(4): 544-51^{(\mathrm{z})}\end{array}$ & $\begin{array}{l}\text { Ações de en- } \\
\text { fermagem no } \\
\text { acompanhamento } \\
\text { do crescimento e } \\
\text { desenvolvimento } \\
\text { infantil de crianças } \\
\text { menores de cinco } \\
\text { anos na UBS em } \\
\text { Franca-SP. }\end{array}$ & Até 5 anos. & $\begin{array}{l}\text { 1)Transmissão de } \\
\text { informação sobre } \\
\text { imunização, aleita- } \\
\text { mento materno e } \\
\text { teste do pezinho, } \\
\text { 2)Orientações indi- } \\
\text { viduais e grupais; } \\
\text { 3)Avaliação da } \\
\text { antropometria, } \\
\text { administração de } \\
\text { medicações, ati- } \\
\text { vidades de pré e } \\
\text { pós-consulta. }\end{array}$ & $\begin{array}{l}\text { Descritivo - } \\
\text { exploratório/ } \\
\text { Brasil }\end{array}$ & 2,6 & VI \\
\hline
\end{tabular}

Bases de Dados: (x) Scopus; (y) Pubmed; (z) Lilacs.

\section{REFERÊNCIAS}

1. Ministério da Saúde Brasil. Secretaria de atenção à saúde. Departamento de ações programáticas estratégicas. Manual para utilização da caderneta de saúde da criança. Brasília (DF): 2005.

2. Ministério da Saúde Brasil. Saúde da criança: acompanhamento do crescimento e desenvolvimento infantil. Brasília, 2002. (Série Cadernos de Atenção Básica, n. 11).

3. Marcondes E, Setian N, Carraza FR. Desenvolvimento físico (crescimento) e funcional da criança. En: Marcondes E. et al. Pediatria básica. 9na ed. São Paulo: Sarvier; 2002.

4. Zeferino AMB, Barros-Filho AA, Bettiol $\mathrm{H}$, Barbieri MA. Monitoring growth. J Pediatr (Rio J) 2003; 79(Supl.1): S23-S32.

5. Silva RRF, Silveira MLM, Giorge AH, Puccini RF. Desenvolvimento. In: Puccini RF, Hilário MOE. Semiologia da criança e do adolescente. Rio de Janeiro: Guanabara Koogan; 2008.

6. World Health Organization (WHO). Ottawa Charter for Health Promotion; 1986.

7. World Health Organization (WHO). Primary health care: report of the international conference primary health care, 
Alma Ata; 1978.

8. Don Nutbeam. Health promotion glossary. Health Promotion International 1998; 13(4): 349-364.

9. Public Health Agency of Canadá. Core Competencies; 2007.

10. Mendes KDS, Silveira RCCP, Galvão CM. Revisão integrativa: método de pesquisa para a incorporação de evidências na saúde e na enfermagem. Texto contextoenferm. 2008; 17(4): 758-764.

11. Ursi ES. Prevenção de lesões de pele no perioperatório: revisão integrativa da literatura [dissertação de mestrado]. [Ribeirão Preto]: Escola de Enfermagem de Ribeirão Preto, Universidade de São Paulo; $2005.130 \mathrm{p}$.

12. Melnyk BM, Fineout-Overholt E. Evidence-based practice in nursing \& healthcare: A guide to best practice. Philadelphia, PA: Lippincott Williams \& Wilkins; 2005.

13. Naidoo J, Wills J. Theory into practice. Cap 1. En: Wills J; Naidoo J. Public health and health promotion - developing practice. Ed: Bailliere Tindall; 2005.

14. Wakimizu R, Fujioka H, Yoneyama A. Empowerment process for families rearing children with developmental disorders in Japan. Nurs Health Sci. 2010; 12, 322-328.

15. Shi L, Zhang J, Wang Y, Caulfield LE, Guyer B. Effectiveness of an educational intervention on complementary feeding practices and growth in rural China: a cluster randomised controlled Trial. $\mathrm{Pu}-$ blic Health Nutr. 2009, 13(4), 556-565.

16. Kramer MS, Matush L, Bogdanovich N, Aboud F, Mazer B, Fombonne E, et al.
Health and development outcomes in 6.5-y-old children breastfed exclusively for 3 or 6 mo. Am J Clin Nutr 2009; 90: 1070-4.

17. Tyler DO, Horner SD. Collaborating With Low-Income Families and Their Overweight Children to Improve Weight-Related Behaviors: An Intervention Process Evaluation. J Spec Pediatr Nurs. 2008; 13(4).

18. Berry D, Savoye M, Melkus G, Grey M. An intervention for multiethnic obese parents and overweight children. Appl Nurs Res. 2007; 20: 63-71.

19. Reifsnider E, Keller CS, Gallagher M. Factors Related to Overweight and Risk for Overweight Status Among Low-Income Hispanic Children. J Pediatr Nurs. 2006; 21:3.

20. Kilanowski JF, Ryan-Wenger N. Health status in an invisible population: carnival and migrant worked children. West J Nurs Res. 2007; 29(1): 100-120.

21. Peña YF, Almeida MCP, Duranza RLC. The nursing work process in care for healthy children at a social security institution in Mexico. Rev Lat Am Enfermagem, 2006; 14(5): 651-7.

22. Berlin A, Hylander I, Törnkvist L. Primary Child Health Care Nurses' assessment of health risks in children of foreign origin and their parents - a theoretical model. Scand J Caring Sci. 2008; 22; 118-127.

23. Figueiredo GLA, Mello DF. A prática de enfermagem na atenção à saúde da criança em unidade básica de saúde. Rev Lat Am Enfermagem 2003; 11(4): 544-51. 